\title{
Observations of 14 young open star clusters with the HEGRA system of Cherenkov telescopes
}

\author{
F. Aharonian ${ }^{1}$, A. Akhperjanian ${ }^{7}$, M. Beilicke ${ }^{4}$, K. Bernlöhrr ${ }^{1}$, H.-G. Börst ${ }^{5}$, H. Bojahr ${ }^{6}$, O. Bolz ${ }^{1}$, T. Coarasa ${ }^{2}$,
} J. L. Contreras ${ }^{3}$, J. Cortina ${ }^{2}$, S. Denninghoff ${ }^{2}$, M. V. Fonseca ${ }^{3}$, M. Girma ${ }^{1}$, N. Götting ${ }^{4}$, G. Heinzelmann ${ }^{4}$, G. Hermann ${ }^{1}$, A. Heusler ${ }^{1}$, W. Hofmann ${ }^{1}$, D. Horns ${ }^{1}$, I. Jung ${ }^{1}$, R. Kankanyan ${ }^{1}$, M. Kestel ${ }^{2}$, A. Konopelko ${ }^{1}$, H. Kornmeyer ${ }^{2}$, D. Kranich ${ }^{2}$, H. Lampeit ${ }^{4,10}$, M. Lopez ${ }^{3}$, E. Lorenz ${ }^{2}$, F. Lucarelli ${ }^{3}$, O. Mang ${ }^{5}$, H. Meyer ${ }^{6}$, R. Mirzoyan ${ }^{2}$, A. Moralejo ${ }^{3}$, E. Ona-Wilhelmi ${ }^{3}$, M. Panter ${ }^{1}$, A. Plyasheshnikov ${ }^{1,8}$, G. Pühlhofer ${ }^{1,12}$, R. de los Reyes ${ }^{3}$, W. Rhode ${ }^{6}$, J. Ripken ${ }^{4}$, G. Rowell ${ }^{1}$, V. Sahakian ${ }^{7}$, M. Samorski ${ }^{5}$, M. Schilling ${ }^{5}$, M. Siems ${ }^{5}$, D. Sobzynska ${ }^{2,9}$, W. Stamm ${ }^{5}$, M. Tluczykont ${ }^{4,11}$, V. Vitale ${ }^{2}$, H. J. Völk ${ }^{1}$, C. A. Wiedner ${ }^{1}$, and W. Wittek ${ }^{2}$

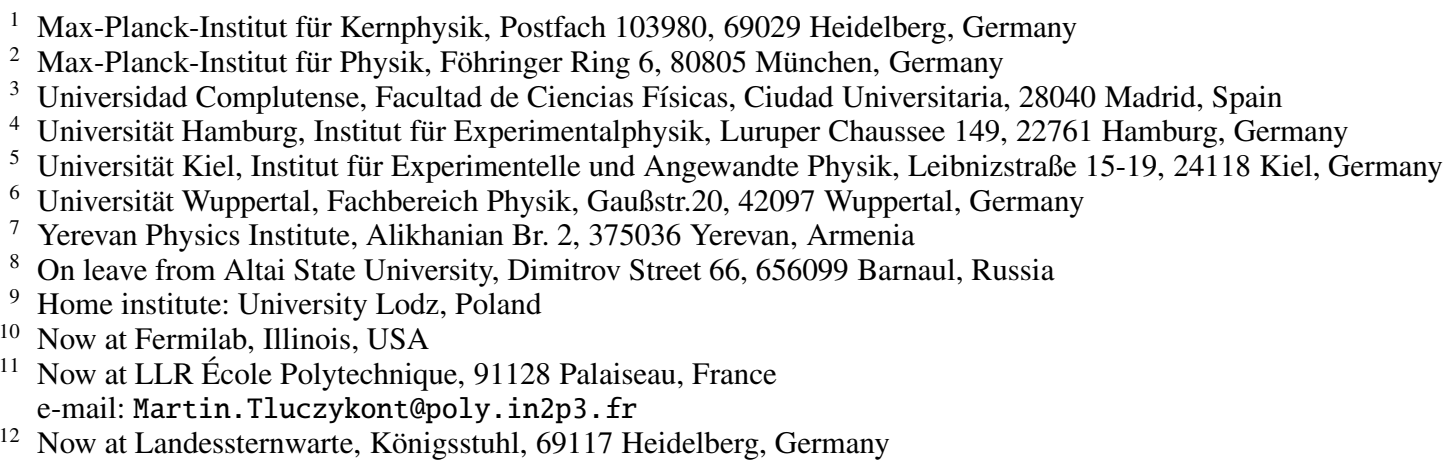

Received 13 April 2005 / Accepted 20 February 2006

ABSTRACT

\begin{abstract}
Context. A sample of 14 young open star clusters has been observed in the TeV energy regime with the stereoscopic system of the HEGRA (High Energy Gamma-Ray Astronomy) Cherenkov telescopes from 1997 to 2002, resulting in more than 300 h of observation time.

Aims. Young open star clusters may contribute to the acceleration of cosmic rays. The detection of $\gamma$-rays (from decaying $\pi^{0} \mathrm{~s}$ produced in hadronic interactions) from these objects could be evidence for such a contribution. The results of our observations are compared to available $\gamma$-ray data and to a simple hadronic model in the framework of shock front acceleration of cosmic rays in the stellar winds of the cluster members to test the potential of the presently available data on young open star clusters to constrain this type of model. Methods. The stereoscopic system of HEGRA Cherenkov telescopes makes use of the atmospheric imaging technique. Air showers initiated by primary Gamma-Rays are recorded as elliptical images in the telescope cameras. The images from the different telescopes are then superimposed to reconstruct the parameters of the primary particle. This technique (stereoscopy) was pioneered by the HEGRA experiment.

Results. No significant excess has been found in the analysed data set of young open star clusters. The derived upper limit on the $\mathrm{TeV}$ gamma-ray flux from Berkeley 87 and the available EGRET data from the same direction do not allow us to fully constrain the simple hadronic model used here. The comparison of the upper limits derived for all 14 objects with the flux detected from $\mathrm{TeV} \mathrm{J} 2032+4130$ (under the assumption of an association of the TeV-signal with the compact stellar association Cyg OB2) suggests that $\gamma$-ray emission from young open star clusters as an object class cannot be ruled out.
\end{abstract}

Key words. gamma rays: observations - cosmic rays - Galaxy: open clusters and associations: general Galaxy: open clusters and associations: individual: Berkeley 87

\section{Introduction}

The question concerning the origin of charged Galactic cosmic rays and their acceleration mechanisms still remains unsolved. Shell-type supernova remnants are commonly considered to be the best candidates for the acceleration of cosmic rays (Lagage \& Cesarsky 1983). In fact, results such as the detection of TeV $\gamma$-rays from the shell-type supernova remnant Cas-A by the HEGRA Cherenkov telescopes (Aharonian et al. 2001), as well as results on RXJ 1713.7-3946 from the CANGAROO and the HESS collaborations (Muraishi et al. 2000; Aharonian et al. 2004) seem to confirm this scenario. However, it is by no means clear that the energy output of the supernova remnants detected in $\gamma$-rays so far is sufficient to explain the observed flux of charged Galactic cosmic rays. An alternative or additional contribution to the cosmic ray acceleration in shell-type supernova remnants is the shock front acceleration in strong stellar winds of massive stars (Montmerle 1979; Casse \& Paul 1980; 
Völk \& Forman 1982; White \& Chen 1992). It was shown that the maximum energy attainable by a particle through shock front acceleration in stellar winds is higher than the maximum energy reached in the shock front of a supernova remnant under similar local conditions (Césarsky \& Montmerle 1983). Young open star clusters contain many young massive stars with up to $60 M_{\odot}$, high mass loss rates, and stellar winds of typically $1000-2000 \mathrm{~km} \mathrm{~s}^{-1}$ (up to $5000 \mathrm{~km} \mathrm{~s}^{-1}$ in particular cases). These objects could thus contribute to the observed flux of charged Galactic cosmic rays via shock front acceleration in the stellar wind of an individual member star or the interaction of the winds of all members of the cluster with each other or the interstellar medium (Polcaro et al. 1991; Manchanda et al. 1996). In this scenario, the accelerated hadronic particles would interact with the interstellar medium or with dense molecular clouds, thus producing neutral pions, which subsequently decay into $\gamma$ rays with energies reaching up to the $\mathrm{TeV}$ energy range and beyond. The recent discovery of the unidentified $\mathrm{TeV}$ source $\mathrm{TeV} \mathbf{J} 2032+4130$ in the vicinity of Cyg OB2 by the system of HEGRA Cherenkov telescopes (Aharonian et al. 2002b), as well as a new unidentified $\mathrm{GeV} / \mathrm{TeV}$ source detected by HESS (Beilicke et al. 2004) that could possibly be associated with the stellar association Cen OB1 (McClure-Griffiths et al. 2001), has renewed interest in the potential of the class of young open star clusters for the acceleration of cosmic rays and for $\gamma$-ray emission in the $\mathrm{TeV}$ energy regime. In this paper, the results of observations of 14 young open star clusters carried out with the stereoscopic system of HEGRA Cherenkov telescopes are presented and compared to the flux observed from TeV J2032+4130. The results obtained for the young open star cluster Berkeley 87 and the spectrum measured by the EGRET experiment from the same direction are used to constrain a simple hadronic model for $\gamma$-ray emission based upon the scenario described above.

\section{The HEGRA Cherenkov telescope system}

From 1996 to 2002, the HEGRA Collaboration has operated a system of five imaging air Cherenkov telescopes (IACTs) on the Canary Island of La Palma $\left(28.75^{\circ} \mathrm{N}, 17.90^{\circ} \mathrm{W}\right)$, at an altitude of $2200 \mathrm{~m}$ a.s.l. The telescopes were used in the stereoscopic observation mode (Daum et al. 1997). Additionally, one telescope (not used for the present analysis) was operated in stand-alone mode (Mirzoyan et al. 1994). The mirror dish of each system telescope consisted of 30 single mirrors with a diameter of $60 \mathrm{~cm}$ each, resulting in a total mirror area of $8.5 \mathrm{~m}^{2}$. Each telescope was equipped with a camera consisting of 271 photomultiplier tubes (pixels) mounted in the focal plane of the mirror dish ( $5 \mathrm{~m}$ focal length) with a total field of view $(\mathrm{FoV})$ of $4.3^{\circ}$. The HEGRA IACT system was operated on an event-by-event basis at an energy threshold of $0.5 \mathrm{TeV}$ for photons of vertical incidence, with energy and angular resolutions of $\Delta E / E=10-20 \%$ and $0.1^{\circ}$, respectively. The pioneering application of the stereoscopic observation technique results in an improvement of sensitivity and $\gamma$-hadron separation in comparison to that of a single telescope. The performance of the HEGRA system of Cherenkov telescopes was reported in detail by Pühlhofer et al. (2003).

\section{HEGRA observations}

The data used in this paper were taken from different observations performed over a long period of HEGRA operation, from 1997 to 2002, resulting in a total exposure of more than $300 \mathrm{~h}$, after removal of observations compromised by bad weather or technical problems. This time corresponds to one third of a year of continuous HEGRA observations. All observations containing at least one of the 14 young open star clusters analyzed here in their FoV were taken into account. These observations include a part of the Galactic plane scan data (Aharonian et al. 2002a), the data on the supernova remnant Cas-A (Aharonian et al. 2001), observations of the region near the COS-B source CG 135+1, the Orion-Nebula (M 42), and dedicated observations of the young open star clusters Berkeley 87 and IC 1805.

Of these star clusters, ten are included in a list of 34 Galactic open clusters by Manchanda et al. (1996) considered as promising canditates for the acceleration of cosmic rays by stellar winds. Two objects from this list, Berkeley 87 and IC 1805, were chosen for dedicated observations with the HEGRA Cherenkov telescopes. Berkeley 87 is the most prominent candidate among the objects of this list. The strong stellar wind $\left(5200 \mathrm{~km} \mathrm{~s}^{-1}\right.$, see Polcaro et al. 1991, and references therein) of one of its member stars, Wolf-Rayet star WR 142, the presence of a compact HII region $(\mathrm{G} 75.77+0.34)$ inside the cluster, and the association with the EGRET source 2EG J2019+3719 (also 3EG J2021+3716) make Berkeley 87 a good candidate for the acceleration of cosmic rays by stellar winds (Polcaro et al. 1991). Additionally, predictions for a $\gamma$-ray flux from Berkeley 87 by Giovanelli et al. (1996), as well as earlier observations with the HEGRA AIROBBIC array (Prahl 1999), have motivated our observations. The second object dedicated observations were made for, IC 1805 , is similar in many aspects to Berkeley 87 and also qualifies as a good candidate for $\gamma$-ray emission (Manchanda et al. 1996).

The unidentified TeV source TeV J2032+4130 was serendipituously discovered in different HEGRA observations dedicated to other objects (Aharonian et al. 2002b). This new TeV source may be associated with the stellar association Cyg OB2 ${ }^{1}$, also figuring in the candidate list of Manchanda et al. (1996).

An air shower initiated by a primary $\gamma$-ray or hadronic particle generates an elliptically shaped image in each triggered camera. Since each telescope has a different viewing angle relative to the shower axis, a complete geometrical reconstruction of the air shower is possible with an image analysis of at least two telescopes. The procedure of technical and weather quality cuts to the data before reconstruction of the events and the image reconstruction criteria are described in Aharonian et al. (2004). In this analysis, at least three remaining images are required in each event for the reconstruction of direction and shower core impact position of the primary particle, as well as the $m s c w$-parameter (mean scaled width), used for $\gamma$-hadron separation. This improves the quality of the reconstruction, the angular resolution, and the $\gamma$-hadron separation.

For each data set, a $3^{\circ} \times 3^{\circ}$ skymap divided into $35 \times 35$ grid search points is produced. Event numbers counted in grid search points from different data sets that correspond to the same astronomical object are merged to obtain the total result for this object. Additionally, the analysis of all grid search points gives a means of testing its quality (see Fig. 2). For each grid point, all events within an angular distance of $0.126^{\circ}$ and with mscw $<$ 1.1 are counted as on-source events $\left(N_{\text {on }}\right)$. The cut on the angular distance for point-like emission was optimized using data of the well-known source Crab Nebula. In case of an extension of the object larger than the event-by-event angular resolution

1 Throughout this paper, we assume this association to hold true and use the results obtained by Aharonian et al. (2002b) for comparison with the results obtained in this work for the other young open star clusters. 


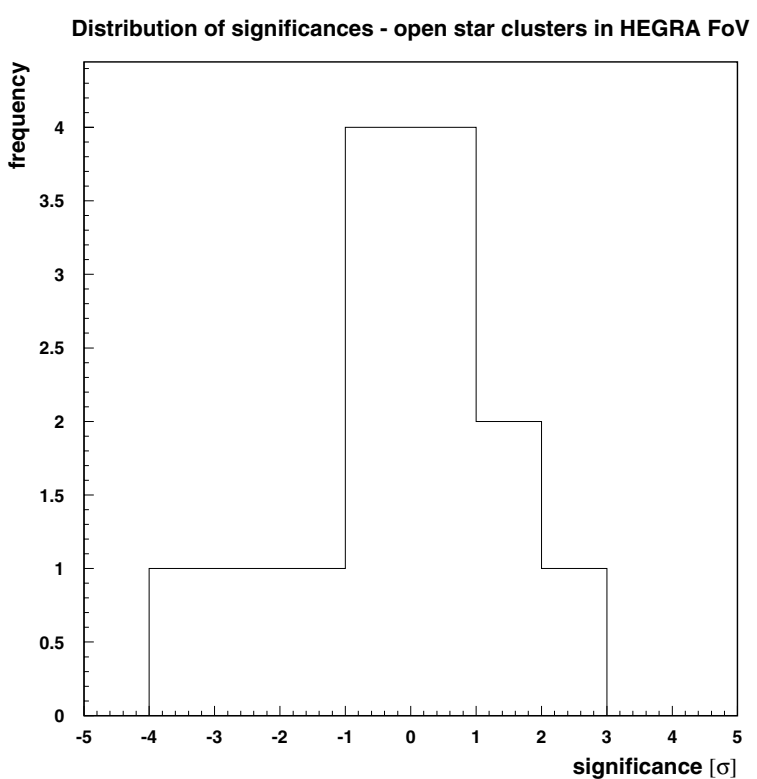

Fig. 1. Distribution of the significance of each of the 14 young open star clusters analyzed in this work. No significant excess was found. This distribution does not contain the significance of the detection

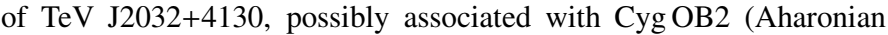
et al. 2002b).

of the telescope system, the cut on the angular distance is adjusted accordingly. Cut optimizations were done with MonteCarlo simulations, assuming a circular homogeneous emission region with an extension identical to the observed physical extension of the cluster, as given by Mermilliod (1995). For each bin of the skymaps, all events within the same angular distance are counted as background events $\left(N_{\text {off }}\right)$, as for the on-events but with $1.3<\mathrm{mscw}<1.5$. The number $N_{\text {off }}$ has to be scaled with the ratio $\alpha$ of on- and off-source region (Li \& Ma 1983), which is different for each observation. A typical value is $\alpha=0.15$. This template-background model is described in detail in Rowell (2003).

For a given object, the data set of each observation $(i)$ containing this object is analyzed separately. Then the event numbers $\left(N_{\text {on }}^{(i)}, \alpha_{\mathrm{i}} N_{\text {off }}^{(i)}\right)$ counted in the grid search points and corresponding to the position of the object are summed up. Since the different observations do not have the same ratios $\alpha$, the significance has to be calculated using the generalized Li \& Ma formula introduced in Aharonian et al. (2004).

For each object, a $\gamma$-rate in Crab-Nebula flux units for identical observational conditions (zenith angle, hardware setup, camera acceptance) is calculated from the data. These measured rates are used to compute upper limits on the integral flux following Helene (1983). Since the objects are located at different positions in the FoV, the individual radial camera acceptances have to be taken into account accordingly.

\section{Results}

The analysis at the positions of all 14 young open star clusters considered in this paper does not yield any significant excess. The distribution of the significance of each of the 14 objects is shown in Fig. 1. The results are summarized in Table 1. For each object, the number of on-events $\left(N_{\text {on }}\right)$ and off-events $\left(N_{\text {off }}\right)$, the observation time, the energy threshold $E_{\mathrm{thr}}$, and the upper limits in units of the Crab-Nebula flux $\left(F_{\mathrm{ul}}\right)$, as well as in units of $10^{-12} \mathrm{~cm}^{-2} \mathrm{~s}^{-1}\left(\Phi_{\mathrm{ul}}\right)$, are given. The values of the calculated
Table 1. Results of the present analysis of the HEGRA data on 14 young open star clusters. Given are the object name, the numbers $\sum_{i} N_{\text {on }}^{(i)}$ and $\sum_{i} \alpha_{i} N_{\mathrm{off}}^{(i)}$, the total observation time, the energy threshold $E_{\mathrm{thr}}$, and the integral flux upper limits $(99 \%$ confidence level) in units of the Crab-Nebula flux $\left(F_{\mathrm{ul}}\right)$ and in units of $\gamma \mathrm{cm}^{-2} \mathrm{~s}^{-1}\left(\Phi_{\mathrm{ul}}\right)$.

\begin{tabular}{|c|c|c|c|c|c|c|}
\hline Object & $\sum_{i} N_{\mathrm{on}}^{(i)}$ & $\sum_{i} \alpha_{i} N_{\mathrm{off}}^{(i)}$ & $\begin{array}{l}\text { Time } \\
{[\mathrm{h}]}\end{array}$ & $\begin{array}{l}E_{\mathrm{thr}} \\
{[\mathrm{TeV}]}\end{array}$ & $\begin{array}{c}F_{\mathrm{ul}} \\
2.6 \sigma \\
{[\mathrm{Crab}]}\end{array}$ & $\begin{array}{l}\Phi_{\mathrm{ul}} / 10^{-12} \\
\text { upper limits } \\
{\left[\gamma \mathrm{cm}^{-2} \mathrm{~s}^{-1}\right]}\end{array}$ \\
\hline Berk 86 & 35 & 34 & 15.5 & 0.88 & 0.05 & 1.02 \\
\hline Berk 87 & 39 & 40 & 13.4 & 0.90 & 0.05 & 1.08 \\
\hline Do29 & 4 & 6 & 22 & 132 & 0.11 & 1.15 \\
\hline extended & 28 & 25 & 2.2 & 1.32 & 0.23 & 2.50 \\
\hline Do3 & 28 & 45 & 15.1 & 0.79 & 0.02 & 0.62 \\
\hline Do33 & 22 & 15 & 3.1 & 1.35 & 0.23 & 2.45 \\
\hline IC 1805 & 119 & 126 & & 118 & 0.03 & 0.39 \\
\hline extended & 352 & 345 & 40.8 & 1.18 & 0.05 & 0.61 \\
\hline Mrk 50 & 169 & 222 & 222.6 & 1.09 & 0.01 & 0.13 \\
\hline NGC 6871 & 9 & 8 & 35 & 079 & 0.14 & 3.36 \\
\hline extended & 39 & 37 & & & 0.20 & 5.05 \\
\hline NGC 6883 & 21 & 20 & & & 0.19 & 4.75 \\
\hline extended & 112 & 115 & 5.5 & 0.19 & 0.28 & 6.89 \\
\hline SgrOB1 & 56 & 56 & 15.1 & 2.89 & 0.05 & 0.17 \\
\hline NGC 1976 & 74 & 73 & 0.7 & 1.07 & 2.95 & 45.00 \\
\hline NGC 1977 & 12 & 3.1 & 0.7 & 1.07 & 1.66 & 25.30 \\
\hline NGC 1980 & 14 & 11.6 & 0.7 & 1.07 & 1.37 & 20.90 \\
\hline NGC 1981 & 13 & 7.5 & 0.7 & 1.07 & 1.50 & 22.90 \\
\hline
\end{tabular}

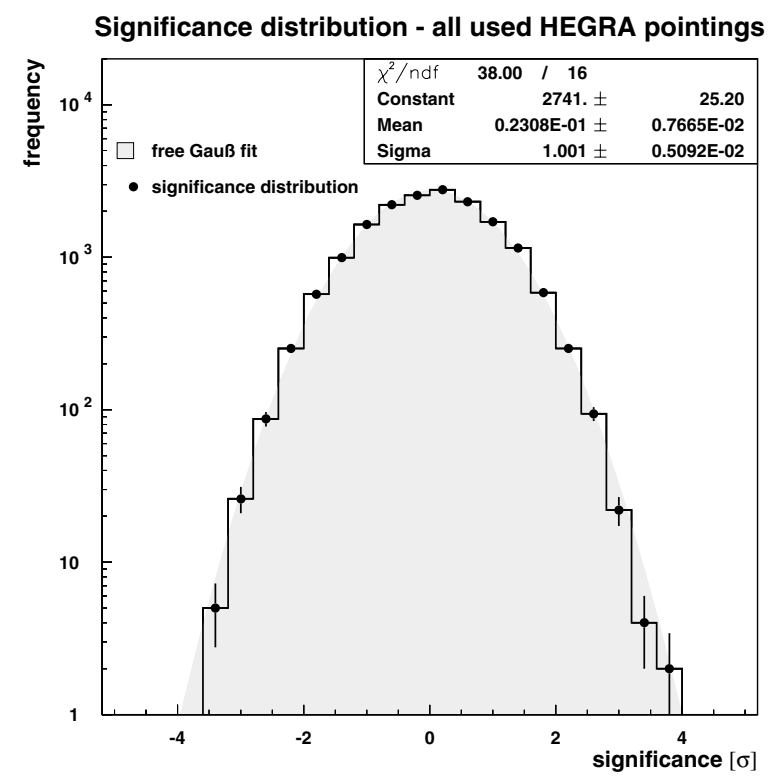

Fig. 2. Distribution of the significances of all skymap grid points of the pointings used for the present analysis (without Cas A and $\mathrm{TeV}$ J2032+4130). The distribution follows the expectation of a standard Gaussian in case of no signal in any field of view.

upper limits are between $0.6 \%$ and $28 \%$ of the Crab-Nebula flux for observation times longer than $1 \mathrm{~h}$. The analysis of objects observed for less than an hour yields upper limits of up to 3 Crab units.

In Fig. 2, a distribution of the significances of all skymapbins in the fields of view of the analyzed data sets, excluding the known signal from the supernova remnant Cas A, is shown. No significant excess is seen. The distribution follows a standard Gaussian of width 1 , demonstrating that the estimation of the significance is very well understood over the whole FoV. 
Table 2. Upper limits $F_{1 \mathrm{TeV}}$ (scaled to $1 \mathrm{TeV}$ and assuming a differential spectral index of 2.0) compared to an expected flux $F_{\text {expected. The }}$ expected flux was calculated assuming that the $\gamma$-ray luminosity of all young open star clusters is similar to the one observed from the unidentified TeV-source TeV J2032+4130, possibly associated with the stellar association Cyg OB2.

\begin{tabular}{lllll}
\hline \hline & Object & $\begin{array}{l}\text { Distance } \\
{[\mathrm{pc}]}\end{array}$ & $\begin{array}{l}F_{\text {expected }} \\
{[\text { Crab] }}\end{array}$ & $\begin{array}{l}F_{1 \mathrm{TeV}} \\
{[\mathrm{Crab}]}\end{array}$ \\
\hline \multirow{4}{*}{$\begin{array}{l}\text { Extreme wind } \\
\text { velocities }\end{array}$} & CygOB2 & $\mathbf{1 7 0 0}$ & $\mathbf{0 . 0 2 6}$ & $=\mathbf{0 . 0 2 6}$ \\
& Berk 87 & 633 & 0.146 & $<0.045$ \\
& Berk 86 & 1112 & 0.060 & $<0.044$ \\
& Do3 & 1820 & 0.022 & $<0.016$ \\
& IC 1805 & 1886 & 0.016 & $<0.035$ \\
\hline & SgrOB1 & 578 & 0.225 & $<0.145$ \\
& Do33 & 1500 & 0.033 & $<0.311$ \\
& NGC 6871 & 1574 & 0.030 & $<0.111$ \\
& NGC 6883 & 2090 & 0.017 & $<0.150$ \\
& Mrk 50 & 2114 & 0.016 & $<0.011$ \\
& Do29 & 2730 & 0.008 & $<0.145$ \\
\hline
\end{tabular}

\section{Discussion}

Although no excess of TeV $\gamma$-rays has been detected from the 14 objects observed, the upper limits obtained still allow for a contribution of the class of young open star clusters to the acceleration of cosmic rays. In this context, it is instructive to compare the upper limits with the flux detected from the unidentified TeV-source TeV J2032+4130, tentatively associating this source with the compact stellar association Cyg OB2, assuming that this object and the observed $\mathrm{TeV}$-flux are typical for a young open star cluster. For this comparison, the upper limits are calculated for a threshold of $1 \mathrm{TeV}$, assuming a spectral index of $\alpha=2$, and then compared to the flux of TeV J2032+4130 scaled to the distance of the corresponding object, as given by WEBDA (Mermilliod 1995) and Manchanda et al. (1996).

This rough calculation gives an estimate of the order of magnitude of an expected emission, given the above assumptions. The results are shown in Table 2. Clusters with member stars having extreme wind velocities are listed in the upper part of this table. In the lower part of Table 2, the results for all other objects also included in the list of Manchanda et al. (1996) are given. Most upper limits (Col. 4) are close to the predicted values (Col. 5). Of all the upper limits, 5 are higher than the expected flux values, and 4 are slightly lower. A strong discrepancy is seen for only one object, Berkeley 87, where the upper limit lies a factor of 4 below the expected value. However, one has to note that the $\gamma$-ray luminosity was assumed to be identical for all open star clusters for the sake of simplicity. Thus, given the variation of the emissivity of the individual objects, the hypothesis of young open star clusters being $\gamma$-ray emitters as a class is not contradicted by these results.

Predictions for the integral flux in the $\mathrm{TeV}$ energy regime expected from Berkeley 87 have been made, based on the assumption of the acceleration of cosmic rays in shock fronts produced by the interaction of the strong stellar winds of the cluster members. In the framework of a simple hadronic model of $\pi^{0}$-decay, scaled to the integral EGRET satellite data of 2EG J2019+371, predictions for different parameters of the model were proposed (Giovanelli et al. 1996). These predictions were rejected in an earlier analysis of the HEGRA data on Berkeley 87 (Tluczykont 2000). To constrain the parameter space in a more quantitative way, we have adopted the model

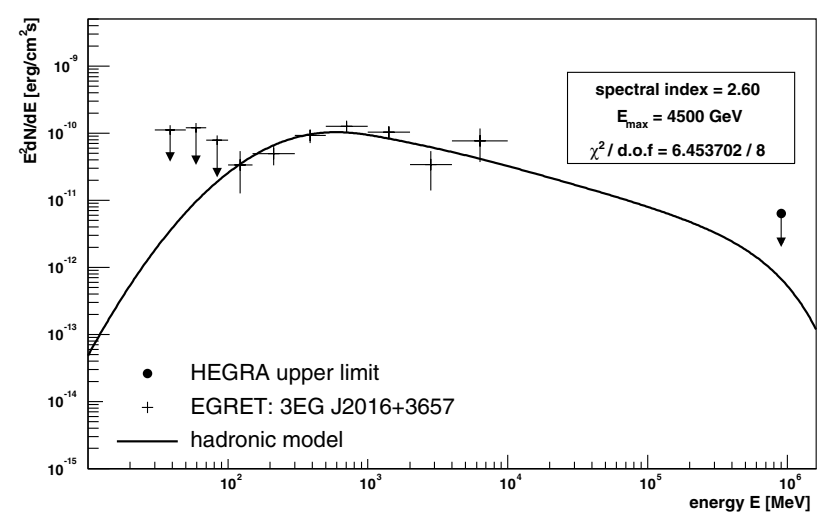

Fig. 3. Example of a fit of the model adopted from Badhwar et al. (1977), Stephens \& Badhwar (1981), and Giovanelli et al. (1996) to the data of the EGRET source 3EG J2016+3657, and the upper limit on Berkeley 87 derived in this work. Here, the parameters $\alpha=2.6, E_{\max }=$ $4500 \mathrm{GeV}$ were used. The $\chi^{2}$-value of the fit is used to derive a confidence level for these parameters (cf. Fig. 4).

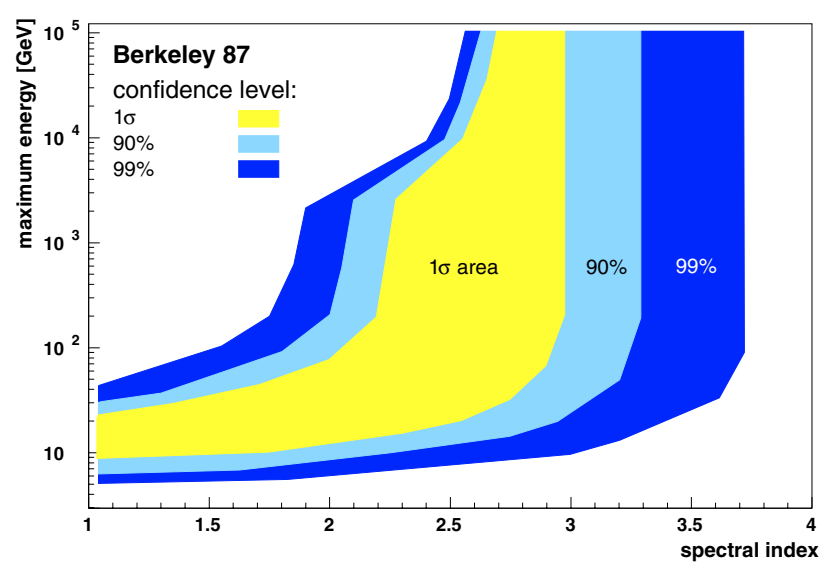

Fig. 4. Confidence level areas for the compatibility of model and data in the parameter plane of $\alpha$ and $E_{\max }$ for Berkeley 87. The confidence levels were derived from the $\chi^{2}$-values of 5000 model fits, using different sets of parameters, to the data as illustrated for one set of parameters in Fig. 3.

used by Giovanelli et al. (1996) with the parametrization of the total inclusive cross section for the production of neutral pions taken from Badhwar et al. (1977). Stephens \& Badhwar (1981) made predictions for a certain set of parameters and fit these predictions to the differential EGRET and HEGRA data; the integral flux upper limit derived in this work was converted into a differential value, given a certain model spectrum. This model is not intended to present a comprehensive treatment of hadronic acceleration in stellar winds, but rather to test the potential of the present observations to constrain this type of models. The only model parameters used here are those determining the shape of the predicted emission, i.e., the spectral index $\alpha$ of the initial hadron spectrum (the accelerated cosmic rays) and the maximum (discrete) energy $E_{\max }$ of the accelerated hadrons. The parameters determining the overall flux intensity (such as the density of ambient matter) are not used here. Instead, the flux intensity is adjusted to fit the available data. Thus, for a given pair of parameters $\left(\alpha, E_{\max }\right)$ a differential energy spectrum can be calculated 
and fit to the EGRET and HEGRA results. In Fig. 3, an example of a fit using one specific set of parameters $\left(\alpha=2.6\right.$ and $E_{\max }=$ $4500 \mathrm{GeV}$ ) is shown. A confidence level can be derived from the $\chi^{2}$ value of the fit for any pair of parameters $\alpha$ and $E_{\max }$. In Fig. 4, the allowed parameter space resulting from a scan over 5000 values of $\alpha$ and $E_{\max }$ is shown for confidence levels (C.L.) of $1 \sigma, 90 \%$, and $99 \%$. The present observations do not allow us to constrain the $E_{\max }$-parameter of the considered model for all values of $\alpha$. Observations with higher sensitivity and lower energy thresholds could significantly constrain the type of model described here (Tluczykont 2003).

\section{Summary}

With the exception of the detection of a $\mathrm{TeV}$ signal in the vicinity of the compact stellar association Cyg OB2 reported earlier (Aharonian et al. 2002b, 2005), no significant excess could be found in the HEGRA data of young open star clusters. Considering the flux upper limits obtained in this work, a contribution to the acceleration of cosmic rays from young open star clusters in the framework of stellar wind acceleration models is still possible. For one object, Berkeley 87, the data of the EGRET and HEGRA experiments have been used to partly constrain the parameter space of a simple $\pi^{0}$-decay model. We conclude that the sensitivity level of the presently available data is not high enough to seriously constrain hadronic type models. Future observations of $\gamma$-rays in the $\mathrm{GeV} / \mathrm{TeV}$ energy regime with instruments of higher sensitivity, together with measurements in the $\mathrm{MeV} / \mathrm{GeV}$ regime, could further constrain such models and possibly answer the question of the contribution of young open star clusters to the acceleration of cosmic rays.

Acknowledgements. The support of the German Federal Ministry for Research and Technology BMBF and of the Spanish Research Council CICYT is gratefully acknowledged. G.R. acknowledges receipt of a von Humboldt fellowship. We thank the Instituto de Astrofísica de Canarias (IAC) for the use of the HEGRA site at the Observatorio del Roque de los Muchachos (ORM) and for supplying excellent working conditions on La Palma. This research has made use of the SIMBAD database, operated at CDS, Strasbourg, France.

\section{References}

Aharonian, F. A., Akhperjanian, A., Barrio, J., et al. (HEGRA collaboration) 2001, A\&A, 370, 112

Aharonian, F. A., Akhperjanian, A. G., Beilicke, M., et al. (HEGRA collaboration) 2002a, A\&A, 395, 803

Aharonian, F. A., Akhperjanian, A., Beilicke, M., et al. (HEGRA collaboration) 2002b, A\&A, 393, L37

Aharonian, F. A., Akhperjanian, A., Beilicke, M., et al. (HEGRA collaboration) 2004, A\&A, 421, 529

Aharonian, F. A., Akhperjanian, A., Beilicke, M., et al. (HEGRA collaboration) 2005, A\&A, 431, 197

Aharonian, F. A., Akhperjanian, A. G., Aye, K.-M., et al. (HESS collaboration) 2004, Nature, 432, 75

Badhwar, G. D., Golden, R. L., \& Stephens, S. A. 1977, Phys. Rev. D - Part. Fields, 15, 820

Beilicke, M., Khelifi, B., Masterson, C., de Naurois, M., \& Schlenker, S. 2004, in Proc. of the 2nd International Symposium on High Energy Gamma-Ray Astronomy, Heidelberg, ed. F. A. Aharonian, \& H. J. Völk, 347

Casse, M., \& Paul, J. A. 1980, ApJ, 237, 236

Césarsky, C., \& Montmerle, T. 1983, Space Sci. Rev., 36, 173

Daum, A., Hermann, G., Heß, M., et al. 1997, Astropart. Phys., 8, 1

Giovanelli, F., Bednarek, W., \& Karakula, S. 1996, J. Phys. G: Nucl. Part. Phys., 22,1223

Helene, O. 1983, Nucl. Instr. Meth., 212, 319

Lagage, P. O., \& Cesarsky, C. J. 1983, A\&A, 118, 223

Li, T. P., \& Ma, Y. Q. 1983, ApJ, 272, 317

Manchanda, R. K., Polcaro, V. F., Norci, L., et al. 1996, A\&A, 305, 457

McClure-Griffiths, N. M., Dickey, J. M., Gaensler, B. M., \& J., G. A. 2001, ApJ, 562,424

Mermilliod, J.-C. 1995, in Astrophysics and Space Science Library, Information \& On-Line Data in Astronomy, 203, 127

Mirzoyan, R., Kankanian, R., Krennrich, F., et al. 1994, Nucl. Instr. Meth. A, 351,513

Montmerle, T. 1979, ApJ, 231, 95

Muraishi, H., Tanimori, T., Yanagita, S., et al. 2000, A\&A, 354, L57

Polcaro, V. F., Giovanelli, F., Manchanda, R. K., et al. 1991, A\&A, 252, 590

Prahl, J. 1999, Ph.D. Thesis, Fachbereich Physik der Universität Hamburg, dissertation.de

Pühlhofer, G., Bolz, O., Götting, N., et al. 2003, Astropart. Phys., 20, 267

Rowell, G. P. 2003, A\&A, 410, 389

Stephens, S. A., \& Badhwar, G. D. 1981, Ap\&SS, 76, 213

Tluczykont, M. 2000, in High Energy Gamma-Ray Astronomy, ed. F. A. Aharonian \& H. J. Völk, 771 (for the HEGRA Collaboration)

Tluczykont, M. 2003, Ph.D. Thesis, Fachbereich Physik der Universität Hamburg, dissertation.de

Völk, H. J., \& Forman, M. 1982, ApJ, 253, 188

White, R. L., \& Chen, W. 1992, ApJ, 387, L81 